\title{
Study on decrease in the viability of brain tumor cells by ultrasound exposure
}

\author{
Akiko Watanabe*, Sakino Iwashiro, Masatsune Minai, Hiroyuki Nishimura and Shinichi Takeuchi \\ Graduate School of Engineering, Toin University of Yokohama, \\ 1614 Kurogane-cho, Aoba-ku, Yokohama, 225-8502 Japan
}

(Received 28 October 2013, Accepted for publication 6 December 2013)

Keywords: Brain tumor, U-87MG cells, Ultrasound exposure, Cell viability PACS number: 43.35.Wa [doi:10.1250/ast.35.170]

\section{Introduction}

Brain functions should be preserved in brain tumor treatment, because important organs like cerebrum, brainstem etc. exist in the brain and they are essential for sustaining life. Therefore, unlike most of other tumor therapies, it is difficult to ablate a wide area of tissues that include surrounding normal tissues into which tumor cells may spread and infiltrate.

Recently, high-intensity focused ultrasound (HIFU) surgery has been used for brain tumor therapy [1-3]. Coagulative necrosis is induced in the tumor cells by the heat generated from the high-energy of focused ultrasound. This ultrasound therapy has some benefits. For example, it is low invasive therapy because it is a trans-skull irradiation and it can be performed repeatedly unlike radiation therapy. On the other hand, it is considered that the inflammatory reaction caused by necrosis damaged normal cells surrounding tumor cells.

We believe it is possible to suppress the damage to surrounding normal cells by ultrasound induced apoptosis [4-6], since apoptosis does not induce an inflammatory response. We are studying about ultrasound exposure conditions to induce apoptosis cell death to brain tumor cells $[7,8]$. In this paper, we will report ultrasound exposure conditions for decrease in viability of human glioblastoma U-87MG cells.

\section{Materials and methods}

\subsection{Cells and cell culture}

Human glioblastoma cells U-87MG (ATCC Cell Bank) were used in our study. The incidence of glioblastoma is the highest among primary brain tumors. Glioblastoma is classified as grade IV in astorcytoma by World Health Organization (WHO). U-87MG cells were grown as a monolayer on the bottom surface of a commercial cell culture flask. Cell culture medium (Minimum Essential Medium) with $10 \%$ fetal bovine serum and $1 \%$ penicillin-streptomycin were added in the cell culture flask. The cells were incubated at $37^{\circ} \mathrm{C}$ in humidified atmosphere with a $5 \% \mathrm{CO}_{2}$ incubator. A photograph of our cultured U-87MG cells by using a phase-contrast microscope is shown in Fig. 1. The doubling time of our cultured U$87 \mathrm{MG}$ cells measured in our laboratory was approximately 28 hours.

\footnotetext{
*e-mail: tm18b23k@ust.toin.ac.jp
}

\subsection{Standing wave type ultrasound exposure system}

The standing wave type ultrasound exposure system was used for the exposing U-87MG cells to ultrasound in the ultrasound field. A stainless steel vibrating plate (the diameter is $180 \mathrm{~mm}$ ) was equipped on the bottom of a water tank $\left(140 \times 140 \times 170 \mathrm{~mm}^{3}\right)$ via an O-ring. The water tank had a circular shape acoustic window (the diameter is $120 \mathrm{~mm}$ ) on its bottom. Standing wave type ultrasound field was formed in the water tank by a Langevin type transducer which was installed at the center and opposite side of acoustic radiation surface of the vibrating plate as shown in Fig. 2.

U-87MG cells were grown as a monolayer on the inner bottom surface of our fabricated cell culture flask $[9,10]$. The flask was filled with cell culture medium MEM to the depth of $5 \mathrm{~mm}$ from the bottom of the cell culture surface. The photograph of the flask is shown in Fig. 3. The flask was made of hollow cylindrical acrylic resin pipe (the area of cell culture surface is $38.5 \mathrm{~mm}^{2}$ ) and had an acoustic window made of thin polystyrene membrane film. This thin polystyrene film acoustic window (the thickness is $25 \mu \mathrm{m}$, Asahi Kasei Chemicals OPS ${ }^{\circledR}$ ) will not block ultrasound propagation. The flask with cell culture medium MEM and U-87MG cells was put in the water tank of our ultrasound exposure system at the distance of $45 \mathrm{~mm}$ from the surface of vibrating plate and the spatial-peak point for ultrasound as well. The distilled water was filled in the water tank to the depth of $100 \mathrm{~mm}$ from the vibrating plate.

2.3. Acoustic field in ultrasound exposure system

The acoustic field of ultrasound in the water tank of ultrasound exposure system was measured with a needle type hydrophone (HNR-1000, ONDA). Tone burst waves (the number of cycles is 100 cycles and the duty ratio about $0.1 \%$ ) at voltage amplitude of $100 \mathrm{mV}_{0-\mathrm{p}}$ and frequency of $150 \mathrm{kHz}$ from a function generator (AFG3252, Tektronix) was amplified by using a RF power amplifier (2100L, ENI) with gain of $50 \mathrm{~dB}$. Output amplified signals (tone burst wave) were applied to a Langevin type transducer which was installed at bottom surface of water tank. The needle type hydrophone was placed at the distance of $45 \mathrm{~mm}$ from the stainless steel vibrating plate. The ultrasound field (the size of measurement area is $10 \times 10 \mathrm{~mm}^{2}$ ) near center point of our ultrasound exposure system was measured with needle type hydrophone by scanning in $0.2 \mathrm{~mm}$ step. The output voltage from function generator which was set for measurement of the acoustic field, it was set to a value lower than when the ultrasound waves 


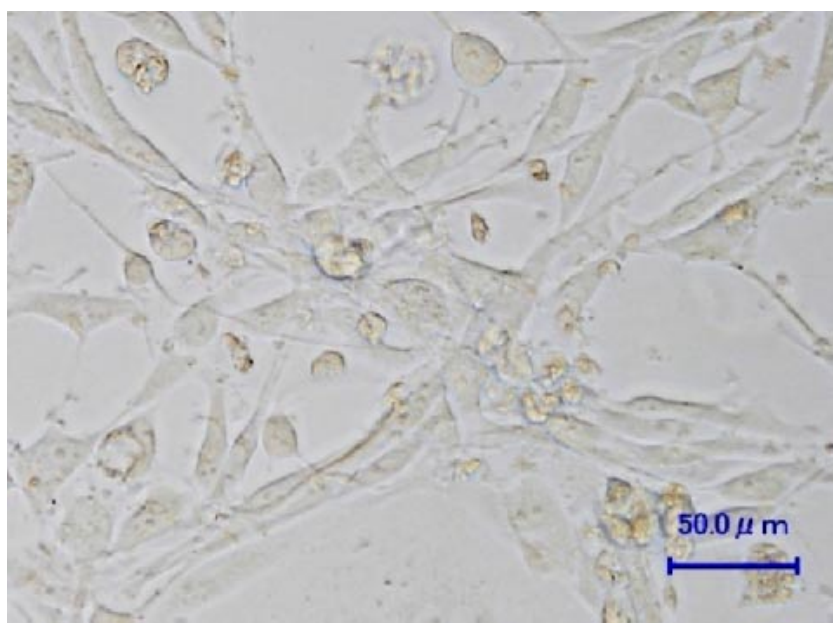

Fig. 1 Photograph of human glioblastoma cells U$87 \mathrm{MG}$ cultured in our laboratory by using a phasecontrast microscope.

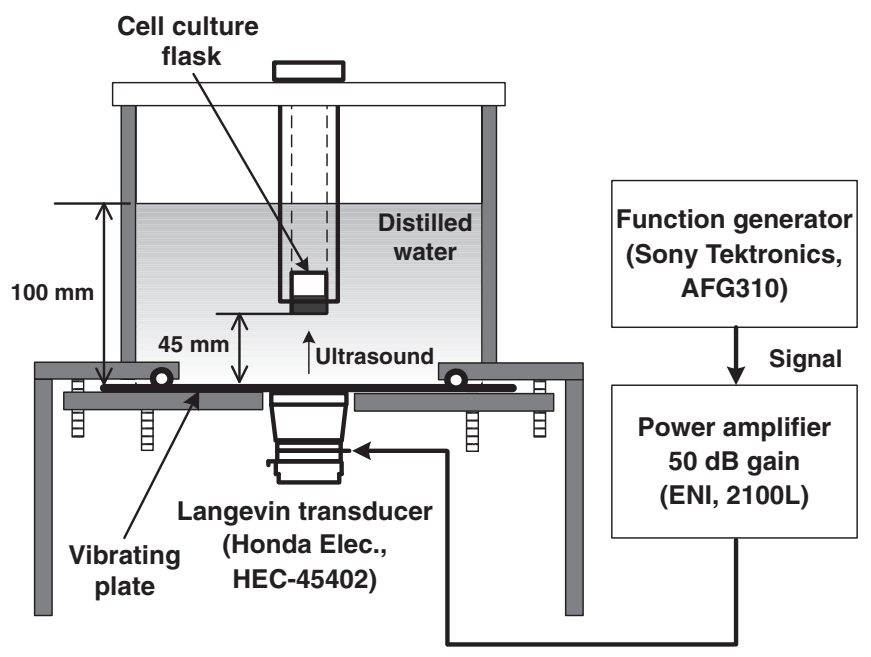

Fig. 2 Schematic diagram of ultrasound exposure system for induction of death to brain tumor cells.
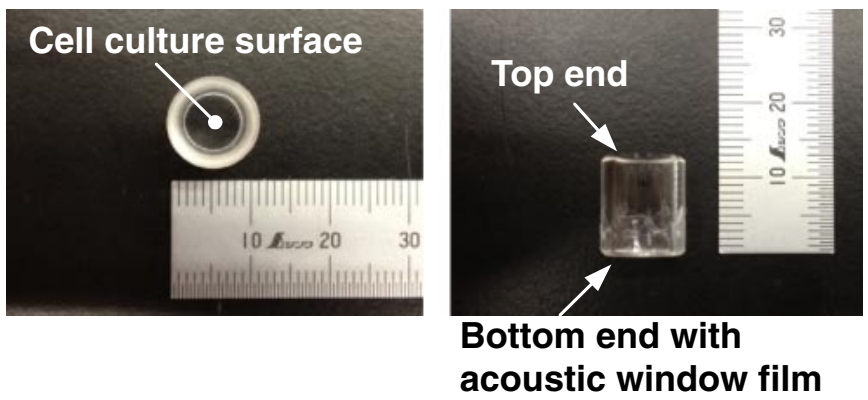

Fig. 3 Our fabricated cell culture flask with acoustic window for ultrasound exposure.

expose to the brain tumor cells. Since the electrode of needle type hydrophone was broken by exposure to high-intensity ultrasound field.

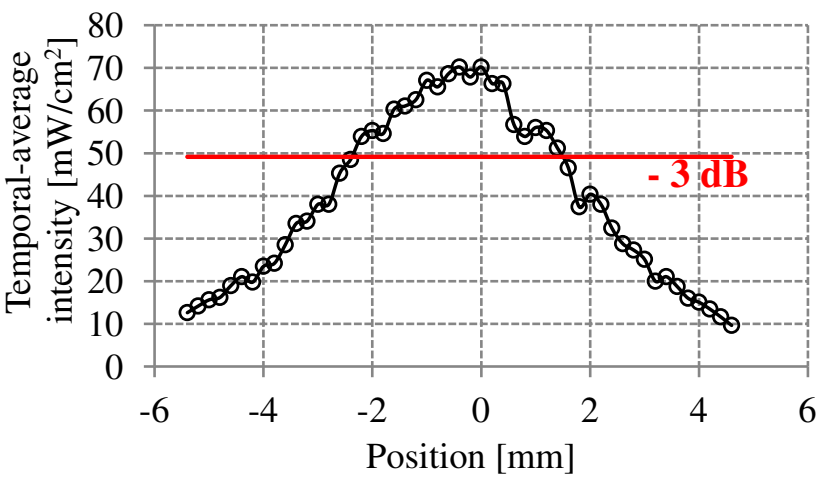

Fig. 4 Intensity of ultrasound near spatial-peak point in water tank of our ultrasound exposure system.

The reference needle type hydrophone (HNR-1000, ONDA) which was calibrated receiving sensitivity was placed at the spatial-peal point and the distance of $45 \mathrm{~mm}$ from the stainless steel vibrating plate in the ultrasound exposure system.

The peak to peak voltage of ultrasound pulse wave at spatial-peak point irradiated from ultrasound exposure system (setting of output voltage from function generator $100 \mathrm{mV}_{0-\mathrm{p}}$ ) was measured by using reference hydrophone. The acoustic intensity at each point of water tank in ultrasound exposure system (the measurement area is $10 \times 10 \mathrm{~mm}^{2}$ ) which was measured by the non-calibrated hydrophone were calculated from the acoustic pressure and the output voltage which was measured by using reference hydrophone. The spatial-peak acoustic intensity when the ultrasound expose to the brain tumor cells was calculated from the multiply spatial-peak acoustic pressure at output voltage from function generator $100 \mathrm{mV}_{0-\mathrm{p}}$ by the rate of increase in output voltage from function generator. The calculation method of acoustic intensity is assumed that the acoustic intensity increase linearly with the output voltage from function generator. The intensity of ultrasound near the spatial-peak point of our ultrasound exposure system is shown in Fig. 4. The $-3 \mathrm{~dB}$ beam area of the measured acoustic field was $10.2 \mathrm{~mm}^{2}$.

2.4. Exposure of U-87MG cells to ultrasound

$\mathrm{U}-87 \mathrm{MG}$ cells (the cell concentration is approximately $2.3 \times 10^{5}$ cells $/ \mathrm{mL}$ ) in our fabricated cell culture flask were incubated at $37^{\circ} \mathrm{C}$ in the humidified atmosphere with a $5 \%$ $\mathrm{CO}_{2}$ incubator for 2 days. U-87MG cells were placed at the water tank of our standing wave type ultrasound exposure system (Fig. 2) and was exposed to continuous ultrasound wave at frequency of $150 \mathrm{kHz}$ and the spatial-peak acoustic intensity of $720 \mathrm{~mW} / \mathrm{cm}^{2}$ for 5 minutes. We prepared a negative control sample for the comparison with a ultrasound irradiated sample. The negative control sample was kept in water at the same temperature for the same time as the ultrasound exposure time. The viability (the ratio of the number of survived cells exposed to ultrasound to the number of negative control cells) of glioblastoma cells was measured by using a Trypan-blue dye exclusion test at every 2 hours after sonication. The adherent cells on the flask were harvested by trypsinization. Both the trypsinized cells and 


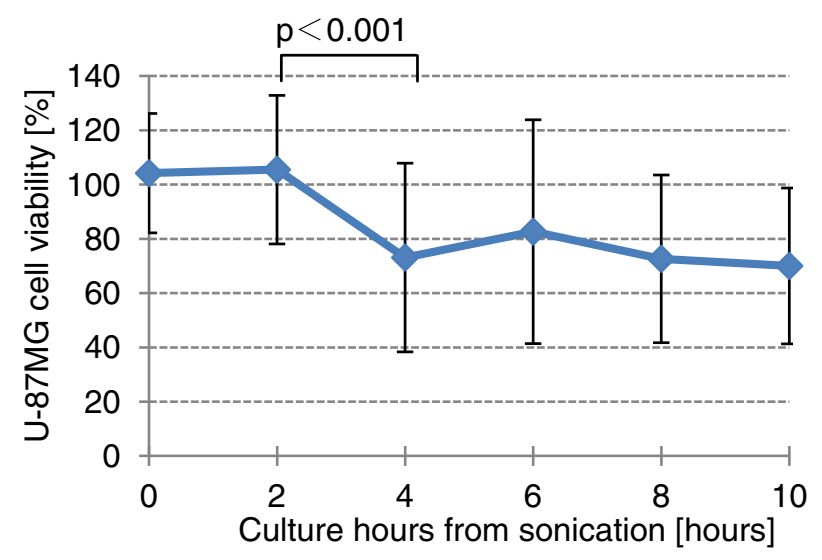

Fig. 5 Relationship between the elapsed time after the ultrasound exposure and the viability of U-87MG cells (the spatial-peak acoustic intensity is $720 \mathrm{~mW} / \mathrm{cm}^{2}$ ).

the floated cells from the bottom surface of the flask by mechanical vibration of ultrasound irradiation were mixed and suspended in centrifuge tube. The cell suspension was mixed with the equal volume of trypan-blue solution and stained. The number of viable cells was counted with using a Burker Turk hemocytometer.

\section{Result and discussion}

Relationship between the elapsed time after the ultrasound exposure and the viability of U-87MG cells is shown in Fig. 5. The viability of sonicated cells was more than $100 \%$ until after 2 hours from sonication. After 4 hours from sonication, cell viability was decreased from $100 \%$ to $80 \%$. The significant differences between cell viability at after 2 hours and 4 hours from sonication were tested. The significant difference was observed in less than $0.1 \%$ significance level. These results indicate that the viability of sonicated cells was decreased from 2 hours to 4 hours after sonication.

Although the conditions of ultrasound exposure, such as the frequency, the spatial-peak acoustic intensity and the ultrasound exposure time, were constant, however, the measurement error of sonicated cell viability was very large. We considered the cause of the large measurement error as follows. The state of the observed U-87MG cells on the bottom surface of the cell culture flask was different for every sonication. Figure 6 shows the change in state of U-87MG cells on the bottom surface of the flask after 5 minute sonication. It can be considered that the effect of 5 minutes sonication on the adhered cells on the bottom surface of the flask differ from those on the floated cells from the bottom surface.

\section{Conclusion}

We measured the effect of exposure to ultrasound at the frequency of $150 \mathrm{kHz}$ and the spatial-peak acoustic intensity of $720 \mathrm{~mW} / \mathrm{cm}^{2}$ on proliferation of brain tumor cells. After 4 hours from sonication, cell viability was decreased. These results indicate that the viability of glioblastoma U-87MG cells was decreased by exposure to ultrasound. (a)
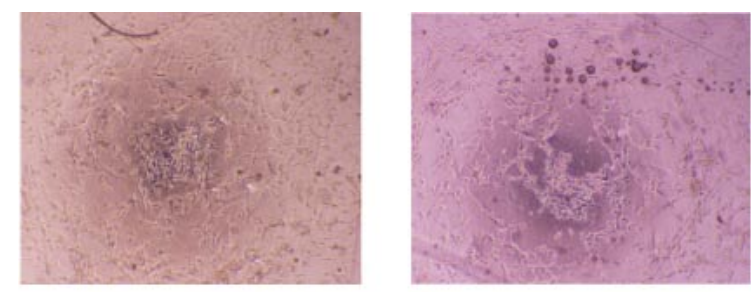

(b)
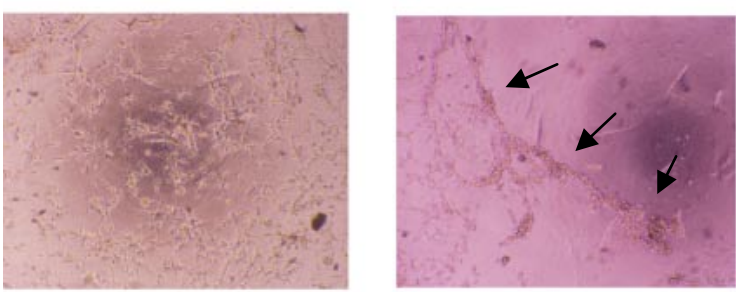

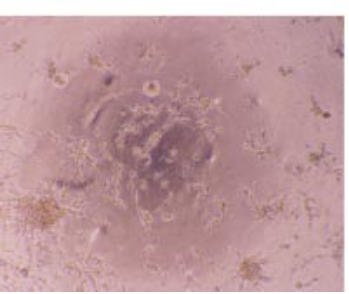

Before sonication

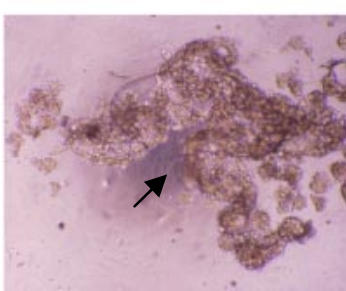

After sonication
Fig. 6 Changes in state of U-87MG cells on the bottom surface of the flask after 5 minutes sonication.

(left) before sonication, (light) after sonication; (a) U87MG cells were adhered on cell culture surface, (b) cells were gathered to one side by sonication, (c) cells were gathered like a sheet and floated from cell culture surface.

\section{Acknowledgement}

I would like to express my gratitude to representative director and president Mr. Kazuo Tadokoro (Koushinseiki Co.) for manufacturing a positioning system for the flask.

\section{References}

[1] InSightec, "Transcranial magnetic resonance-guided focused ultrasound surgery: Current applications and progress for noninvasive neurosurgery," ExAblate 4000 White paper, 1(1), pp. 1-8 (2010).

[2] Y. F. Zhou, "High intensity focused ultrasound in clinical tumor ablation," World J. Clin. Oncol., 2, 8-27 (2011).

[3] E. Martin and B. Werner, "Focused ultrasound surgery of the brain," Curr. Radiol. Rep., 1, 126-135 (2013).

[4] H. Honda, T. Kondo, Q.-L. Zhao, L. B. Feril and H. Kitagawa, "Role of intracellular calcium ions and reactive oxygen species in apoptosis induced by ultrasound," Ultrasound Med. Biol., 30, 683-692 (2004).

[5] L. B. Feril, T. Kondo, Z.-G. Cui, Y. Tabuchi, Q.-L. Zhao, H. Ando, T. Misaki, H. Yoshikawa and S. Umemura, "Apoptosis induced by the sonomechanical effects of low intensity pulsed ultrasound in a human leukemia cell line," Cancer Lett., 221, 145-152 (2005).

[6] Y. Furusawa, Y. Fujiwara, P. Campbell, Q.-L. Zhao, R. Ogawa, M. A. Hassan, Y. Tabuchi, I. Takasaki, A. Takahashi and T. Kondo, "DNA double-strand breaks induced by cavitational mechanical effects of ultrasound in cancer cell lines," Plos one, 7(1), pp. 1-8 (2012). 
A. WATANABE et al.: DECREASE IN BRAIN TUMOR CELL VIABILITY BY US

[7] A. Watanabe, S. Iwashiro, M. Minai, H. Nishimura and S. Takeuchi, "Basic study on induction of cell death of glioblastoma U-87MG by ultrasound exposure," Neurosonology, 25 (Suppl.), 119 (2013).

[8] A. Watanabe, S. Iwashiro, M. Minai, H. Nishimura and S. Takeuchi, "Study on ultrasound irradiation method and condition for induction of cell death to brain tumor cells," Proc. Autumn Meet. Acoust. Soc. Jpn., pp. 1277-1278 (2013).

[9] S. Iwashiro, A. Watanabe, M. Minai, H. Nishimura and S.
Takeuchi, "Development of adherent cell culture flask with acoustic window film for ultrasound exposure," IEICE Tech. Rep., 113(68), US2013-9, pp. 7-12 (2013).

[10] S. Iwashiro, A. Watanabe, Y. Onozuka, M. Minai, H. Nishimura and S. Takeuch, "Fabrication and estimation of cell culture flasks with acoustic windows for culture and ultrasound exposure-comparison of the welding methods of the flask body and acoustic window film-," Proc. Autumn Meet. Acoust. Soc. Jpn., pp. 1279-1280 (2013). 